\title{
Genetic Analysis of Circadian and Ultradian Locomotor Activity Rhythms in Laboratory Rats
}

\author{
Franziska Wollnik, ${ }^{1,3}$ Klaus Gärtner, ${ }^{1}$ and Dietmar Büttner ${ }^{2}$ \\ Received 25 Nov. 1985-Final 20 Oct. 1986
}

\begin{abstract}
Obvious differences exist in the daily activity patterns of the LEW/Ztm and ACI/Ztm inbred strains of laboratory rats. The inbred strain ACII Ztm shows a clear 24-h rhythm of locomotor activity. The activity pattern of the LEWIZtm strain, however, is characterized by ultradian rhythms of 4- and 4.8-h periods. Genetic analysis of crosses between the two strains was used to examine the relative amounts of additive genetic and dominance effects for traits associated with circadian and ultradian rhythms of locomotor activity. The measured variables of the timing mechanism (amplitude c; acrophase $\varphi ; 24,4.8-$, and 4- $h$ spectral estimates) exhibited substantial heritability, with general dominant-recessive modes of inheritance and the associated dominance variance.
\end{abstract}

KEY WORDS: circadian and ultradian rhythms; locomotor activity; classical genetic analysis; laboratory rats.

\section{INTRODUCTION}

Previous locomotor activity studies (Büttner and Wollnik, 1984) identified genetically based differences in the daily patterns of five inbred strains of rats. The amplitude of the 24-h rhythm of locomotor activity of the inbred strain LEW/Ztm was lower than that of other inbred strains, e.g., $\mathrm{ACI} / \mathrm{Ztm}, \mathrm{AS} / \mathrm{Ztm}$, or $\mathrm{BS} / \mathrm{Ztm}$. In addition, male rats of the $\mathrm{LEW} / \mathrm{Ztm}$ stain exhibited typical ultradian rhythms with periods of 4 and $4.8 \mathrm{~h}$. The

This research was supported by Grant SFB 146 from the Deutsche Forschungsgemeinschaft.

${ }^{1}$ Central Animal Laboratory of the Medical School Hannover, D-3000 Hannover 61, F.R.G.

${ }^{2}$ Central Animal Laboratory for Experimental Medicine of the Medical School Essen, D4300 Essen, F.R.G.

${ }^{3}$ To whom correspondence should be addressed at Department of Neurobiology and Physiology, Northwestern University, Evanston, Illinois 60201. 
females displayed no such rhythms (Wollnik, 1985). Previous studies indicated that the sex-related difference in the ultradian rhythms depends on the endogenous estradiol level of the adult animals (Wollnik and Döhler, 1986). The endogenous character of these strain-characteristic patterns was demonstrated by their persistence under continuous-dark conditions.

Genetic analyses of circadian rhythms have been conducted with Drosophila (Konopka and Benzer, 1971), the common bread mold Neurospora (Feldman and Hoyle, 1973), and the green alga Chlamydomonas (Bruce, 1972). Recently, this potentially powerful technique was employed in circadian rhythm studies in mammals. Inbred strain comparisons were used in order to analyze food and water consumption (Possidente and Hegmann, 1980) as well as enzyme activity rhythms in mice (Peleg et al., 1982). The first genetic analysis of parameters associated with the circadian rhythm of body temperature in mice was performed by Connolly and Lynch (1983). This study showed a substantial heritability for traits involved with the timing mechanism.

The basis of the present study describing the mode of inheritance of different variables associated with the circadian and ultradian rhythms of locomotor activity in ACI/Ztm and LEW/Ztm rats was, likewise, a classical genetic analysis.

\section{MATERIALS AND METHODS}

\section{Animals and Maintenance Conditions}

The parental animals were derived from inbred lines, which were continuously monitored by biochemical and immunological methods for isohistocompatibility. Ten initial crosses between $\mathrm{ACI} / \mathrm{Ztm}\left(\mathrm{P}_{1}\right)$ and LEW/Ztm $\left(\mathrm{P}_{2}\right)$ animals resulted in reciprocal $\mathrm{F}_{1}(\mathrm{ACI} \times \mathrm{LEW}$ and LEW $\times \mathrm{ACI}$ ) offspring. The $\mathrm{F}_{1}$ animals were then backcrossed, resulting in all possible backcross matings. Each of the two reciprocal matings consisted of three mated pairs. All rats were weaned at 28 days of age and maintained in genetic and sex-segregated groups. The rats were reared in our laboratory under a light-dark cycle of $12: 12 \mathrm{~h}$ and controlled environmental conditions (room temperature, $22 \pm 1^{\circ} \mathrm{C}$; relative humidity, $55 \pm$ $5 \%$ ). The animals were free of all pathogens specified in the GV-SOLAS (1977) list.

During the course of the measurements the animals were individually kept in polycarbonate cages (Makrolon Type III, $55 \times 33 \times 20 \mathrm{~cm}$ ) on sterile wooden granules. A pelleted diet (Altromin $1324 ; 23 \%$ protein, $5 \%$ fat, $12 \mathrm{~kJ} / \mathrm{g}$ utilizable energy) and tap water rom Makrolon bottles were 
available ad libitum. The rooms were entered once a week for animal maintenance.

\section{Locomotor Activity Measurements}

Locomotor activity was recorded with an electronic movement analyzer, operating on a capacitant system (Plonait et al., 1982), which supplies a signal proportional to the horizontal movement of the animal. The output was recorded at 10 -s intervals by a microcomputer (Apple II). The parents and $F_{1}$ generations were observed between August 1983 and February 1984 . Each parent and $F_{1}$ generation consisted of approximately 12 male and 12 female rats ( $90-150$ days old) randomly selected from an isogenic group. Measurements were made at random in blocks of six identically sexed animals of different genetic background, e.g., three LEW $\delta$ and three $\mathrm{ACI} \delta$ or three $\mathrm{F}_{1}(\mathrm{ACI} \times \mathrm{LEW})$ o and three $\mathrm{F}_{1}(\mathrm{LEW}$ $\times \mathrm{ACI})+$, for 10 consecutive days.

The backcross generations were measured between February 1984 and September 1984. Each possible backcross generation consisted of six males and six females ( $70-170$ days old) randomly selected from the offspring. Locomotor activity measurements of the backcross generations were carried out in 16 blocks of six animals, measured for 6 consecutive days each. Each block consisted, likewise, of identically sexed animals from two different backcross generations, e.g., three females of the $F_{1}$ $(\mathrm{LEW} \times \mathrm{ACI}) \times \mathrm{LEW}$ generation and three females of the $\mathrm{F}_{1}(\mathrm{LEW} \times$ ACI) $\times$ ACI generation.

\section{Data Analysis}

The locomotor activity data for each animal were analyzed by leastsquares cosinor analysis (Halberg et al., 1967; Henkel and Mletzko, 1981) and generalized harmonic spectral analysis (Halberg and Panofsky, 1961; Panofsky and Halberg, 1961).

For a given time series, the cosinor analysis determines the bestfitting cosine function of a specified period, in this case $24 \mathrm{~h}$. Subsequently, individual estimates of the circadian amplitude $c$ and acrophase $\varphi$ were obtained. The amplitude $c$ is the largest positive deviation from the rhythm-adjusted average or mesor and is expressed as a percentage of the 24-h mean. The acrophase is the time lag between a reference point and the crest time of the cosine curve. The acrophase of a fitted cosine function with a period of $24 \mathrm{~h}$ is simply the time of day at which the crest of the cosine function occurs. For genetic analysis, the timing of the thythms was expressed as the phase angle difference $\varphi$ between the ac- 
rophase and lights-off. According to conventional definition the phase angle is negative if the acrophase occurs after lights-off (Aschoff $e t$ al., 1960).

The harmonic spectral analysis evaluates the statistical significance of a given rhythm and allows pooling of spectral estimates of individualsin this case animals of the same genetic group-in order to improve their statistical reliability. The amplitudes $\left(S^{\prime}\right)$ of distinct spectral estimates are a measure of the extent to which oscillations of various period lengths contribute to the total variability of a time series. From this analysis, individual estimates of the amplitude of the 24-, 4.8-, and 4-h spectral estimates were obtained for genetic analysis.

\section{Genetic Analysis}

Genetic analysis of inbred strain crosses was performed according to methods described in detail by Mather and Jinks (1982), including estimates of the mean $m$, additive $[a]$, dominance $[d]$, and three nonallelic, first-order interaction components, $[i],[j]$, and $[l]$, between pairs of genes. The gene effects were classified as being additive and independent in their action, because the joint scaling test devised by Cavalli (1952) indicated that nonallelic interactions were insignificant.

The results of this scaling test are depicted in Table I. The weighted least-squares values for $m,[a]$, and $[d]$ were calculated from the generation means under the assumption of an absence of nonallelic interactions. The weights used are the reciprocals of the squared standard deviations of each generation mean. The squared deviations of the expected and observed generation means are distributed as a $\chi^{2}$ with $(n-3)$ degrees of freedom, where $n$ is the number of observed generations. If $\chi^{2}$ proves insignificant, the $m,[a]$, and $[d]$ model is judged to be adequate and nonallelic interactions to be absent.

The ratio $[d] /[a]$, the so-called "potence ratio" (Mather and Jinks, 1982), is a measure of the relative potence of the parental gene sets. The potence ratio can theoretically take any value between zero and infinity. While a significant potence ratio indicates dominance of the individual genes predominantly in the same direction, zero potence does not necessarily indicate absence of dominance.

Estimates of genetic and environmental variance components (Table II) were obtained by second-degree statistics as described in detail by Mather and Jinks (1982). The environmental variance is represented by the variance of the isogenic generations $\left(V_{\mathrm{E}}=V_{\mathrm{P} 1}=V_{\mathrm{P} 2}=V_{\mathrm{F} 1}\right)$. Because no $\mathrm{F}_{2}$ data were available, it was not possible to separate additive $V_{\mathrm{A}}$ and dominance $V_{\mathrm{D}}$ variance. Values for $V_{\mathrm{A}}+V_{\mathrm{D}}=V_{\mathrm{B} 1}+V_{\mathrm{B} 2}-$ 


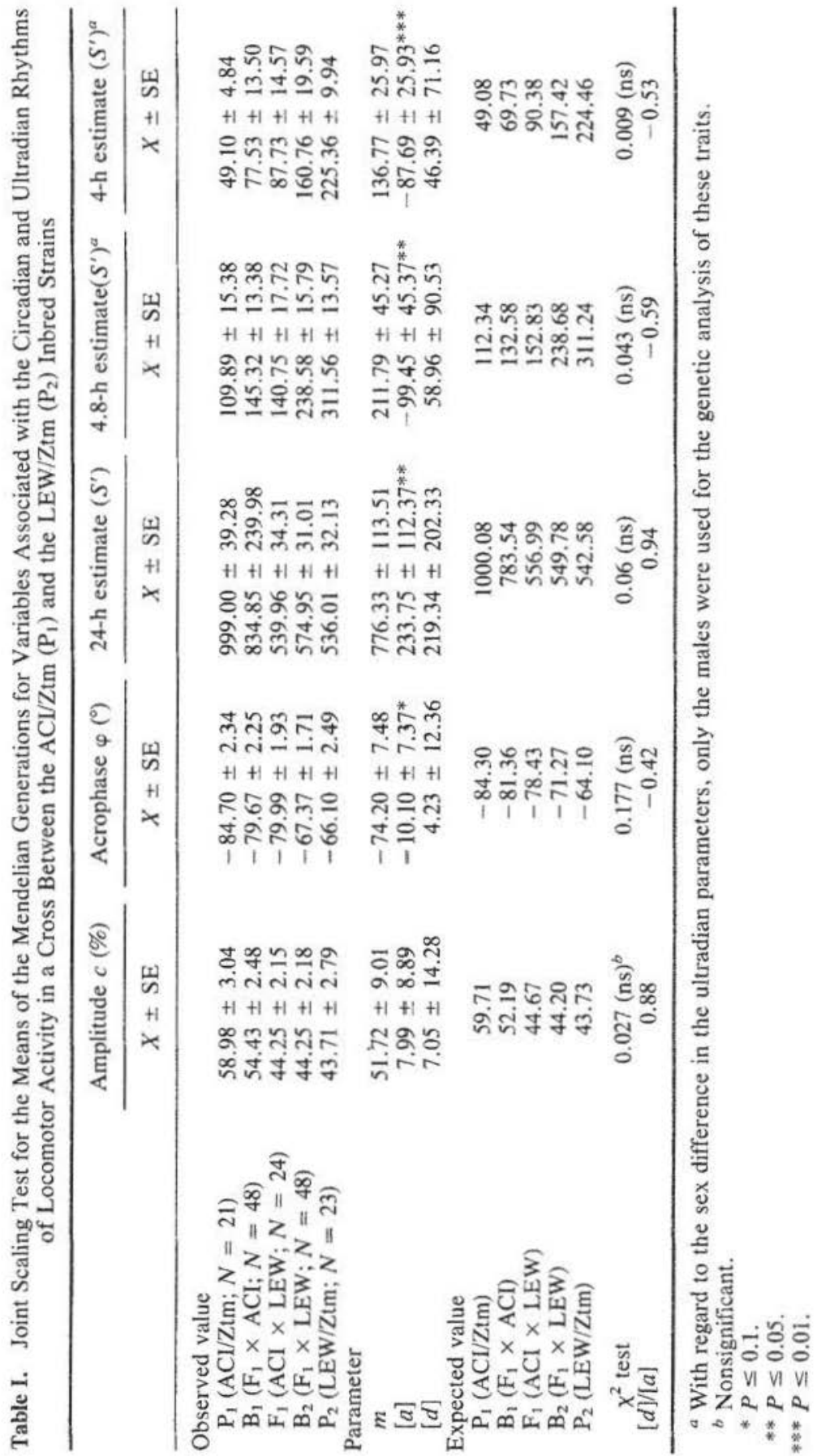


Table II. Estimates of Environmental $\left(V_{E}\right)$, Additive and Dominance Variances $\left(V_{\mathrm{A}}+\right.$ $V_{\mathrm{D}}$ ), and Broad-Sense Heritability $\left(h^{2}\right)$ from Genetical Analysis of Crosses Between ACI/ $\mathrm{Ztm}\left(\mathrm{P}_{1}\right)$ and LEW/Ztm $\left(\mathrm{P}_{2}\right)$ Inbred Rats for Variables Associated with the Circadian and Ultradian Rhythms of Locomotor Activity

\begin{tabular}{|c|c|c|c|c|c|}
\hline & $\begin{array}{c}\text { Amplitude } \\
c \\
(\%)\end{array}$ & $\begin{array}{c}\text { Acrophase } \\
\varphi \\
\left({ }^{\circ}\right)\end{array}$ & $\begin{array}{c}\text { 24-h } \\
\text { estimate } \\
\left(S^{\prime}\right)\end{array}$ & $\begin{array}{c}\text { 4.8-h } \\
\text { estimate } \\
\left(S^{\prime}\right)^{a}\end{array}$ & $\begin{array}{c}\text { 4-h } \\
\text { estimate } \\
\left(S^{\prime}\right)^{a}\end{array}$ \\
\hline \multicolumn{6}{|l|}{ Variance } \\
\hline $\mathrm{P}_{1}(\mathrm{ACI} / \mathrm{Ztm} ; N=21)$ & 193.54 & 115.03 & 32591.08 & 4964.61 & 491.51 \\
\hline $\mathrm{B}_{1}\left(\mathrm{~F}_{1} \times \mathrm{ACI} ; N=48\right)$ & 294.51 & 243.98 & 76747.28 & 8598.85 & 8749.73 \\
\hline $\mathrm{F}_{1}(\mathrm{ACI} \times \mathrm{LEW} ; N=24)$ & 111.31 & 89.68 & 28253.91 & 7534.24 & 5092.25 \\
\hline $\mathrm{B}_{2}\left(\mathrm{~F}_{1} \times \mathrm{LEW} ; N=48\right)$ & 228.74 & 139.71 & 46155.37 & 11972.74 & 18414.49 \\
\hline $\mathrm{P}_{2}(\mathrm{LEW} / \mathrm{Ztm} ; N=23)$ & 179.30 & 142.56 & 23746.81 & 4232.80 & 2271.48 \\
\hline$V_{\mathrm{E}}$ & 148.86 & 109.24 & 28211.43 & 6066.47 & 3236.87 \\
\hline $\begin{array}{c}V_{\mathrm{A}}+V_{\mathrm{D}}=2\left(V_{\mathrm{B} 1}+V_{\mathrm{B} 2}\right) \\
-4 V_{\mathrm{E}}\end{array}$ & 223.52 & 165.22 & 66479.80 & 8438.65 & 20690.48 \\
\hline Heritability, $h^{2}$ & 0.60 & 0.60 & 0.70 & 0.58 & 0.86 \\
\hline
\end{tabular}

${ }^{a}$ With regard to the sex difference in the ultradian parameters, only the males were used for the genetic analysis of these traits.

$2 V_{E}$ were, therefore, estimated. Broad-sense heritability was estimated as $h_{2}=\left(V_{\mathrm{A}}+V_{\mathrm{D}}\right) /\left(V_{\mathrm{A}}+V_{\mathrm{D}}+V_{\mathrm{E}}\right)$.

\section{RESULTS}

\section{Parent and $F_{1}$ Generations}

Figures $1 \mathrm{~A}-\mathrm{C}$ show the daily patterns of locomotor activity of the two parental and the $\mathrm{F}_{1}$ generations as well as the best-fitting cosine function of these data. Figures $2 \mathrm{~A}-\mathrm{C}$ depict the spectral analyses with spectral estimates for 24-, 4.8-, and 4-h periods. Means and standard errors of all variables in each generation are given in Table I, together with the joint scaling test devised by Cavalli (1952). No variable showed significant differences between the two reciprocal $F_{1}$ or the four reciprocal backcross generations (Mann-Whitney $U$-test), so values obtained for the individuals of each generation were pooled. In addition, no significant differences between males and females of each generation were observed with regard to variables associated with the 24-h rhythm (amplitude $c$, acrophase $\varphi$ and 24-h spectral estimate). These values were, therefore, also pooled. Genetic analysis of the ultradian variables (4- and 4.8-h spectral estimates) was based on values obtained for the males only.

Previous studies indicated significant differences in the daily locomotor activity pattern of the ACI/Ztm and LEW/Ztm inbred strains (Büttner and Wollnik, 1984; Wollnik, 1985). This difference is supported by 


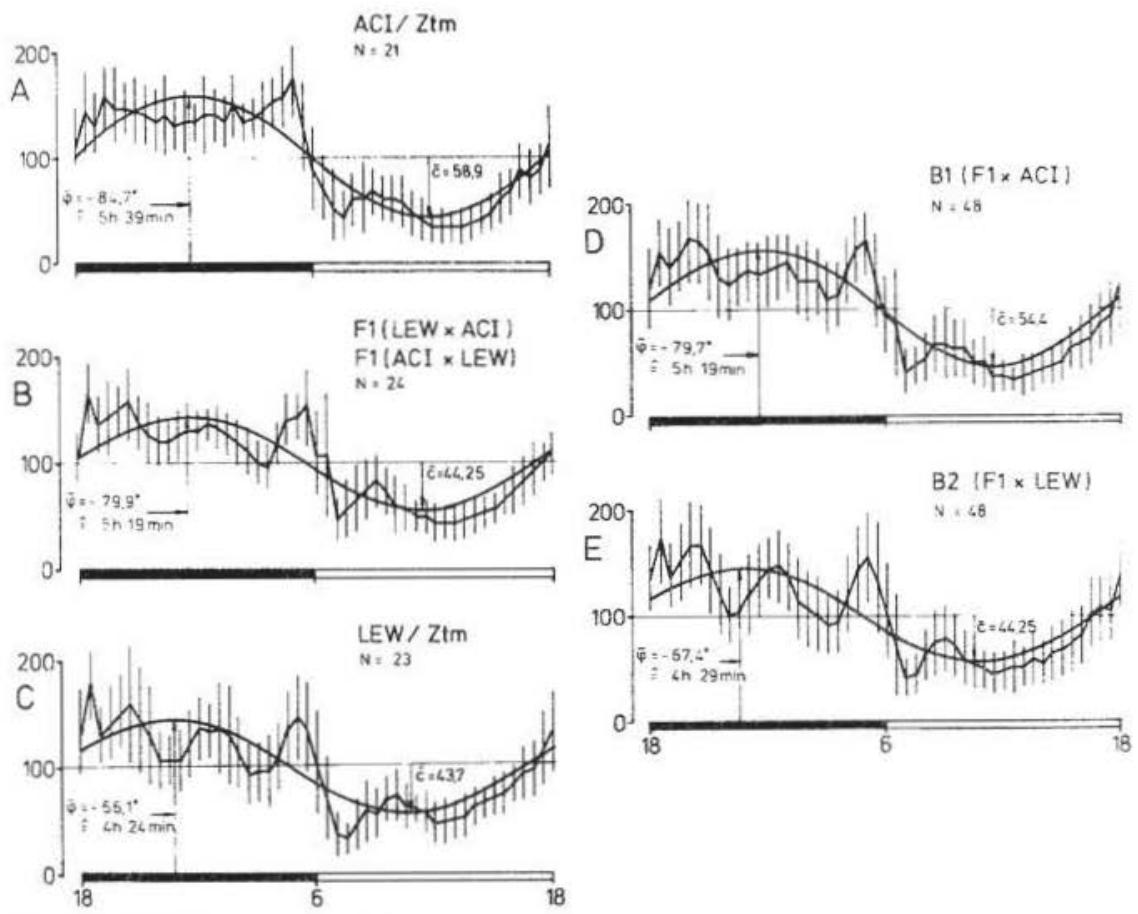

Fig. 1. Daily locomotor activity (LA) patterns of the Mendelian generations under a lightdark regime of $12: 12 \mathrm{~h}$, expressed as a percentage of the 24-h mean. Standard deviations between animals are plotted as vertical lines. Abcissa units: 30-min averages pooled for $N$ males and females of each generation, whereby the first 30-min average is replotted at the end. The best-fitting cosine function of the 24 -h period was analyzed by least-squares cosinor analysis (Halberg et al., 1967; Henkel and Mletzko, 1981) and the mean acrophase ( $\bar{\varphi})$ and mean amplitude $(\bar{c})$ where calculated from the individual estimates by forming mean vectors. The filled portion of the horizontal bar indicates the dark period.

present information showing the amplitude of the best-fitting cosine function for the $\mathrm{ACI} / \mathrm{Ztm}$ rats $(\bar{c}=58.98 \%)$ as being much higher than that for the $\mathrm{LEW} / \mathrm{Ztm}$ rats $(\bar{c}=44.25 \%)$. The $\mathrm{F}_{1}$ generation $(\bar{c}=44.25 \%)$ displayed a strong dominance toward the LEW/Ztm parent. Similar relationships were found for the 24-h spectral estimates.

The acrophase of the locomotor activity rhythm of the $\mathrm{ACI} / \mathrm{Ztm}$ strain $(\dot{\varphi}=-84.70=5 \mathrm{~h} 39 \mathrm{~min})$ appeared more than $1 \mathrm{~h}$ later than in the LEW/Ztm strain $(\tilde{\varphi}=-66.10=4 \mathrm{~h} 24 \mathrm{~min})$. The acrophase value of the $F_{1}$ generation $(\bar{\varphi}=-79.99=5 \mathrm{~h} 19 \mathrm{~min})$ lay between those of the parents.

Amplitudes in the 4.8- and 4-h spectral estimates were significant only in the LEW/Ztm strain. The amplitudes of the ultradian spectral 


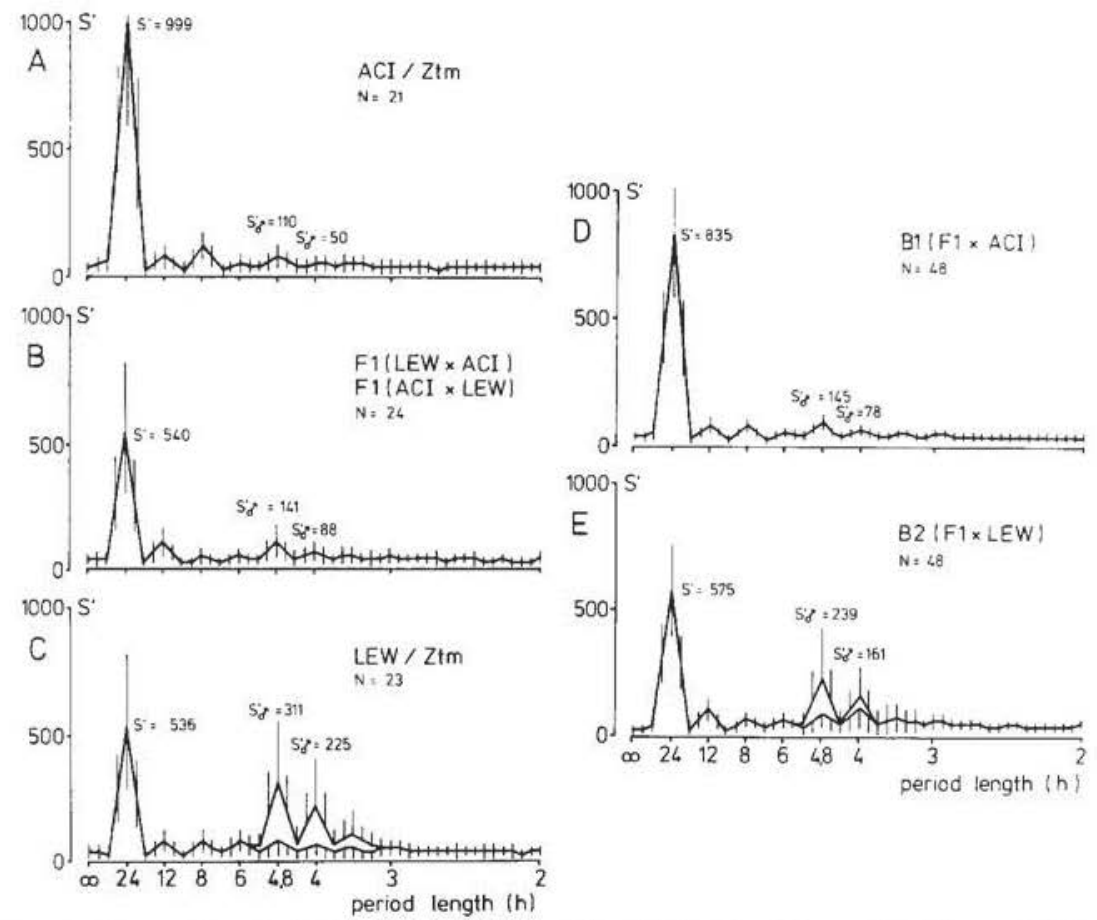

Fig. 2. Spectral analysis (Halberg and Panofsky, 1961; Panofsky and Halberg, 1961) of locomotor activity of the Mendelian generations under a light-dark regime of 12:12 h. In the case of the LEW/Ztm parents (C) and the LEW-backcross generation (E), the 4.8- and 4-h spectral estimates are plotted separately for the males (upper line) and females (lower line) in order to show the sex difference in these traits. All other spectral estimates were pooled for males and females. The $95 \%$ limits of the spectral estimates are plotted as vertical lines.

estimates of the $\mathrm{ACI} / \mathrm{Ztm}$ strain did not differ significantly from the baseline. The $F_{1}$ generation showed a repeated strong dominance toward the nonultradian ACI/Ztm strain for both variables. Differences between the reciprocal $F_{1}$ generations were not observed in any variable. The investigated strain differences are, apparently, not influenced by sex-linked or maternal effects.

\section{Backcross Generations}

The daily patterns of locomotor activity and the spectral analyses of the backcross generations are shown in Figs. 1D and E and Figs. 2D and $\mathrm{E}$, respectively. Because of the time lag between testing of the isogenic and testing of the backcross generations, effects of environmental differ- 
ences cannot be excluded. However, deviation of the expected and observed values was not sufficient in any case to cause a significant $\chi^{2}$ in the joint scaling test (Table I). The weighted least-squares estimates of $m,[a]$, and $[d]$ are, therefore, acceptable and nonallelic interactions are negligible. The additive component $[a]$ was significant in nearly all traits. Dominance $[d]$ was, on the other hand, not significant.

The potence ratio $[d] /[a]$ for the 24 -h estimate and the amplitude $c$ were positive and greater than zero. From this it is evident that there is a majority of dominant genes in the low-amplitude strain, i.e., in the LEW/ $\mathrm{Ztm}$ strain. In contrast, the potence ratio of the acrophase $\varphi$ and the 4.8and 4-h spectral estimates is negative, which means that there is a preponderance of dominant genes for these traits in the nonultradian ACI/ Ztm strain.

\section{Genetic Estimates}

Environmental $\left(V_{\mathrm{E}}\right)$, additive and dominance variance $\left(V_{\mathrm{A}}+V_{\mathrm{D}}\right)$, and broad-sense heritability $\left(h^{2}\right)$ for the five variables are shown in Table II. All variables showed additive and dominance components of variance and substantial broad-sense heritabilities, indicating that these traits would shift in response to selection.

In the case of the ultradian variables there was some indication that the characteristics might have a single-gene mode of inheritance. Neither the $\mathrm{ACI} / \mathrm{Ztm}$ strain, the $\mathrm{F}_{1}$ generation, nor the $\mathrm{ACI}$ backcrosses displayed ultradian rhythms, whereas the LEW-backcross males exhibited a ratio of 11 animals showing distinct ultradian rhytinms to 13 animals lacking the ultradian component. These numerical proportions are consistent with an expected 1:1 Mendelian ratio for a single-locus, two-allele model with dominance. This hypothesis needs further testing.

\section{DISCUSSION}

Each locomotor activity rhythm parameter measured (amplitude $c$; acrophase $\varphi ; 24-, 4.8-$, and 4-h spectral estimates) exhibited a substantial broad-sense heritability with generally intermediate or low dominance. This observation can be interpreted as identification of traits which apparently are not closely related to fitness but, rather, shift in response to selection (Falconer, 1981).

It is, however, important to note that the use of extreme strains in classical analysis often yields inflated estimates of heritability (Whitney et al., 1970). Comparative analysis of heritability for the 24-h spectral 
estimate against other inbred strains revealed an intraclass correlation coefficient (Festing, 1979) $r_{\mathrm{I}}=0.46$ for this trait.

An initial genetic analysis of circadian rhythm parameters in the body temperature of mice (Conolly and Lynch, 1983), likewise, indicated a substantial heritability for traits involved with the timing mechanism (i.e., amplitude, acrophase). The average body temperature, on the other hand, exhibited a near-zero heritability.

As the comparison of the $F_{1}$ means with the midparent values had already suggested, all variables showed a dominance component of variance. Estimates of the potence ratio $[d] /[a]$ indicated a preponderance of dominance for the amplitude $c$ and the 24-h spectral estimate in the $\mathrm{LEW} / \mathrm{Ztm}$ strain and a preponderance of dominance for the acrophase $\varphi$ and the 4- and 4.8-h spectral estimates in the ACI/Ztm strain. This difference in the dominance pattern does not necessarily imply regulation of the circadian and ultradian rhythmicity by different genes.

The numerical proportions of ultradian and nonultradian animals in the LEW backcrosses suggest that the expression of ultradian rhythms is determined by a single recessive gene locus. Further investigations of other segregating generations must be done to support this hypothesis, because the distinction between a polygenic and a single mode of inheritance is difficult to establish. Wright (1934), for example, studied the mode of inheritance of polydactyly in guinea pigs. Although the segregating generations showed numerical proportions of animals with and without extra digits, implying a recessive single-locus determination, mating of the four-toed "homozygous recessive" segregants back to the recessive parent strain resulted in three-toed offspring in $16 \%$ of the pairings. With a single-locus mode of inheritance such matings should have bred true. Wright's work demonstrated that in certain cases the classical crosses cannot be used to distinguish between a single-locus and a polygenic mode of inheritance. A convincing demonstration of a single-locus determination generally involves breeding from one or more of the segregating generations.

Single-gene mutations affecting circadian rhythms have been isolated in two species of Drosophila (Konopka, 1972) and Neurospora (Feldman and Hoyle, 1973). For example, the per locus in Drosophila (Konopka, 1972) has alleles that are associated with normal, 19-h, and 29-h periods as well as arrhythmicity in the circadian range, and the same locus imposes similar changes in the range of seconds in the period of oscillations in courtship song patterns (Kyriacou and Hall, 1980). In addition, mammalian albinism is known to alter different aspects of circadian rhythms. An investigation of mice homozygous for the recessive albino or pinkeye- 
dilute locus showed shorter circadian rhythms for this mutations than for pigmented controls (Possidente et al., 1982).

In general, the results of this and other studies (Bruce, 1972; Büttner and Wollnik, 1984; Connolly and Lynch, 1983; Feldmann, 1973; Konopka, 1972; Peleg et al., 1982; Possidente and Hegmann, 1980; Possidente et al., 1982) indicate that parameters of daily rhythms in behavioral and physiological variables are genetically influenced in many organisms. For most animals the environment is a complex of variables fluctuating with a distinct 24 -h periodicity. Biological rhythms in the behavior of individual animals can be seen as various strategies designed to cope with the time structure of the environment. These adaptive strategies have at least two aspects. On one hand, they assure an optimal temporal arrangement of animal behavior within the cycles of the environment. On the other hand, this external adaptation results in an internal temporal order which in itself may have selective value.

Due to the significant differences in several characteristics of their locomotor activity rhythms, the LEW/Ztm and $\mathrm{ACI} / \mathrm{Ztm}$ strains were chosen as the parent animals in this study. Only those variables expressed under a LD 12:12 lighting regime were analyzed. We can, therefore, not distinguish between genetic differences in the observed rhythms and genetic differences in the pacemaker driving the rhythm. Further genetic analyses of the free-running periods under constant dark and of phaseresponse curves are necessary to determine whether gene differences exert a direct influence on the circadian pacemaker.

\section{ACKNOWLEDGMENTS}

We thank Miss S. Lühmann for her help and technical assistance and T. R. Geimer, A. Herrmann, R. Kluge, and K. G. Rapp for their comments on the manuscript.

\section{REFERENCES}

Aschoff, J., Klotter, K., and Pohl, H. (1960). Circadian vocabulary. Cold Spring Harbor Symp. Quant. Biol. 25.

Bruce, V. G. (1972). Mutants of the biological clock in Chlamydomonas reinhardi. Genetics 70:537-548.

Büttner, D., and Wollnik, F. (1984). Strain-differentiated circadian and ultradian rhythms in locomotor activity of the laboratory rat. Behav. Genet. 14:137-152.

Cavalli, L. L. (1952). An analysis of linkage in quantitative inheritance. In Reeve, E. C. R., and Waddington, C. H. (eds.), Quantitative Inheritance, London, pp. 135-144.

Connolly, M. S., and Lynch, C. B. (1983). Classicial genetic analysis of circadian body temperature rhythms in mice. Behav. Genet. 13:491-500.

Faiconer, D. S. (1981). Introduction to Quantitative Genetics, 2nd ed., Longman, London. Feldman, J. R., and Hoyle, M. N. (1973). Isolation of circadian clock mutants of Neurospora crassa. Genetics 75:605-613. 
Festing, M. F. W. (1979). Inbred Strains in Biomedical Research, Macmillan, London.

GV-SOLAS (1977). Liste von Erregern zur Spezifizierung bei SPF-Versuchstieren. Veröffentlichungen der Gesellschaft für Versuchstierkunde Nr. 2.

Halberg, F., and Panofsky, H. (1961). I. Thermo variance spectra; Methods and clinical illustrations. Exp. Med. Surg. 19:285-321.

Halberg, F., Tong, Y. L., and Johnson, E. A. (1967). Circadian system phase-An aspect of temporal morphology; Procedures and illustrative examples. In von Mayersbach, $\mathrm{H}$. (ed.), The Cellular Aspects of Biorhythms, Springer Verlag, Berlin.

Henkel, W., and Mletzko, H. G. (1981). Die Cosinor-Darstellung biorhythmischer Zeitreihen und ihre Anwendung bei Belastungsuntersuchungen. Z. ges. Hyg. 27:195-198.

Konopka, R. J. (1972). Circadian Clock Mutants of Drosophila melanogaster, Ph.D. thesis, California Institute of Technology, Pasadena.

Konopka, R. J., and Benzer, S. (1971). Clock mutants of Drosophila melanogaster. Proc. Natl. Acad. Sci. 68:2112-2116.

Kyriacou, C. P., and Hall, J. C. (1980). Circadian rhythm mutations in Drosophila melanogaster. Proc. Natl. Acad. Sci. 77:6729-6733.

Mather, K., and Jinks, J. L. (1982). Biometrical Genetics, 3rd ed., Chapman and Hall, London.

Panofsky, H., and Halberg, F. (1961). II. Thermo variance spectra; Simplified computational example and other methodology. Exp. Med. Surg. 19:323-338.

Peleg, L., Nesbitt, M. N., and Ashkenazi, I. E. (1982). A strain difference in the daily rhythm of glyceraldehyde-3-phosphate dehydrogenase activity in the mouse. J. Comp. Physiol. 148:137-142.

Plonait, H., Büttner, D., and Müschen, U. (1982). Ein kapazitiv arbeitendes System zur Messung der Bewegungsaktivität kleiner Labortiere. Z. Versuchstierkunde 24:244-249.

Possidente, B., and Hegmann, J. P. (1980). Circadian rhythms under common gene control. J. Comp. Physiol. 139:121-125.

Possidente, B., Hegmann, J. P., Carlson, L., and Elder, B. (1982). Pigment mutations associated with altered circadian rhythms in mice. Physiol. Behav. 28:389-392.

Whitney, G., McClearn, G. E., and DeFries, J. C. (1970). Heritability of alcohol preference in laboratory mice and rats. J. Hered. 61:165-169.

Wollnik, F. (1985). Sex differences in the daily pattern of locomotor activity. Naturwissenschaften 72:158-161.

Wollnik, F., and Döhler, K.-D. (1986). Effects of adult or perinatal hormonal environment on ultradian rhythms in locomotor activity of laboratory LEW/Ztm rats. Physiol. Behav. 38:229-240.

Wright, S. (1934). The result of crosses between inbred strains of guinea-pigs, differing in the number of digits. Genetics 19:537-551.

Edited by David Blizard 